\title{
A Convex Hull Formulation for the Design of Optimal Mixtures
}

\author{
Suela Jonuzaj $^{\mathrm{a}}$ and Claire S. Adjiman ${ }^{\mathrm{a},{ }^{*}}$

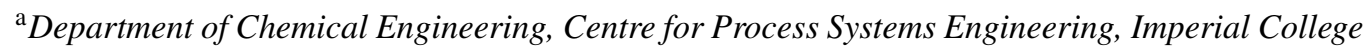 \\ London, South Kensington Campus, London SW7 2AZ, United Kingdom \\ *c.adjiman@imperial.ac.uk
}

\begin{abstract}
The design of mixtures plays an important role in improving process and product performance but is challenging because it requires finding the optimal number, identities and compositions of mixture components and using nonlinear property models. To address this, a general modeling framework for mixture design problems is presented. It integrates Generalized Disjunctive Programming (GDP) into Computer-Aided Mixture/blend Design via Hull Reformulation (HR). The design methodology is applied successfully to a case study involving solid-liquid equilibrium calculations to find an optimal solvent mixture that dissolves ibuprofen. The results show that the proposed GDP-based approach appears very promising for the design of mixture problems. The HR approach is used to solve mixture problems successfully. Its overall computational efficiency is found to be better than that of Big-M approach. Numerical difficulties arising from the absence of components in the final mixture can be avoided, leading to computationally efficient solutions.
\end{abstract}

Keywords: Optimal Mixture Design, Generalized Disjunctive Programming, Hull Reformulation

\section{Introduction}

The design of mixtures is an essential activity across a wide range of chemical engineering applications, from separation processes to product design. Although pure compounds can be used in many applications, they do not always offer the desired performance. Moreover, restrictions arise on the use of common compounds as a result of changing regulations (e.g., REACH regulations). The formulation of mixtures, on the other hand, offers a potential route to enhanced performance, because mixtures can exhibit properties that equal or even surpass those of pure components.

Hence, over the last decades there has been growing interest in the development of systematic methods, within the Computer-Aided Mixture/blend Design $\left(\mathrm{CAM}^{\mathrm{b}} \mathrm{D}\right)$ framework (Achenie et al. 2003 Gani. 2004), for the determination of optimal mixtures. Several methodologies for designing solvent mixtures, blends of refrigerants and polymers have been reported in the literature. Buxton et al. (1999) proposed a systematic decomposition-based procedure to select optimal solvent blends for nonreactive, multicomponent gas absorption processes. Karunanithi et al. (2005) proposed a decomposition-based computer-aided molecular/mixture design methodology which was applied successfully to two solvent design case studies. A Multi-Objective Optimisation approach was developed by Papadopoulos et al. (2013) for obtaining the optimal binary fluid mixtures for organic Rankine cycles. Siougkrou et al. (2014) investigated the design of binary solvent mixtures as part of the conceptual designs of reactive processes. Their approach focused on the design of a $\mathrm{CO}_{2}$-expanded solvent and its impact on process performance. In the area of refrigerants, Duvedi and Achenie (1997) proposed a mathematical programming framework for the design of environmentally-friendly refrigerant mixtures. Vaidyanathan and El-Halwagi (1996) proposed a 
general and systematic methodology for the design of polymer blends, where binary polymer mixtures that match a set of target properties were determined. In a different approach, Solvason et al. (2009) proposed a methodology where property clustering techniques were integrated with the existing mixture design framework to design multicomponent mixtures. Yunus et al. (2014) presented a systematic four-step methodology for the design of blended liquid products.

Two aspects of general mixture design problems make them particularly challenging: the complexity that arises from dealing with a mixture with an unknown number of components and the numerical difficulties that accompany large nonlinear and combinatorial problems. Indeed, the majority of existing methodologies focus on the design of mixtures with a fixed number of components and have been applied mostly to binary mixtures (Buxton et al., 1999; Karunanithi et al. 2005; Papadopoulos et al., 2013; Siougkrou et al. 2014; Duvedi and Achenie, 1997), with some exceptions such as the work of Klein et al. (1992), Solvason et al. (2009) and Yunus et al. (2014) who have presented methodologies for the design of multicomponent mixtures. Furthermore, within the $\mathrm{CAM}^{\mathrm{b}} \mathrm{D}$ framework, the design problems have often been posed as Mixed-Integer Nonlinear Programming (MINLP) problems, where the optimal molecular structure is determined with respect to a set of property constraints. Solving the resulting mixed integer optimization problems can be challenging due to nonconvexities in the space of the continuous variables and a large combinatorial solution space which may lead to several numerical difficulties.

In view of the challenges that remain in the area of formulation design, the main purpose of this work is to create a comprehensive and systematic mathematical programming approach for the solution of the generalised $\mathrm{CAM}^{\mathrm{b}} \mathrm{D}$ problem. We have recently proposed (Jonuzaj et al. 2015) a novel methodology where the number, identity and compositions of mixture constituents are optimised simultaneously. Within this approach, Generalized Disjunctive Programming (GDP) (Raman and Grossmann, 1994) was employed to formulate the CAM ${ }^{b} \mathrm{D}$ problem, in order to address the difficulties arising from the complexity of the model and facilitate the problem formulation. Although GDP has been applied to process network systems, scheduling and distillation column design (Grossmann and Trespalacios, 2013), it had not previously been used in formulating mixture problems. In our initial work, the GDP problems were reformulated as MINLP problems using the Big-M (BM) approach. Here the approach is extended to Hull Relaxations (HR) and a comparison of the relative performance of big-M and HR approaches is presented.

\section{Problem formulations}

The GDP formulations were introduced and described in detail in our recent work (Jonuzaj et al. 2015); we focus here on investigating the use of BM and HR approaches on mixture problems.

The $\mathrm{CAM}^{\mathrm{b}} \mathrm{D}$ problem involves the generic formulation of the mixture design problem where the optimal number of mixture ingredients, the optimal identities of the components and their compositions are identified, such that all given specifications are satisfied and the performance objective is optimised. The mixture to be designed consists of fixed molecules (e.g., solutes) and unknown components (e.g., solvents) that are selected from a predefined list. In order to develop the formulations, we define several index sets. The first is the set of components in the mixture, $I=\left\{1, \ldots, N_{c}\right\}$, where $N_{c}$ is the total number of components. We define $N^{\prime}$ as the number of fixed components and $N_{\max }$ as the maximum number of components to be designed (i.e., the maximum number of components in the mixture is $\left.N_{c}=N^{\prime}+N_{\max }\right) . N=\left\{1, \ldots, N_{\max }\right\}$ is the set of components to be designed and the set $S=\left\{1, \ldots, N_{s}\right\}$ defines the list of compounds from which the unknown mixture components must be chosen.

\subsection{GDP formulation of the $C A M^{\mathrm{b}} D$ problem}

The GDP formulation of the CAM ${ }^{\mathrm{b}} \mathrm{D}$ problem consists of the objective function, $f(x)$, general constraints, $g(x)$, that must hold regardless of the discrete choices, conditional constraints that depend on the discrete choices and are formulated inside disjunctions, and logic relations, $\Omega(Y)$, 
that connect the disjunctive sets. Two different sets of disjunctions are included in the formulation. The first set involves disjunctions for selecting each component from a list of pure compounds; the each compound is assigned through Boolean variables, $Y_{i, s}$ (a compound $s$ is (not) assigned to component $i$ in the mixture if $Y_{i, s}$ is True (False)). The second set involves property constraints that depend on the number of mixture ingredients and that are active when the corresponding Boolean variable $\tilde{Y}_{n}$ is True. The GDP formulation of the mixture problem can be written as:

$$
\begin{aligned}
& \min _{x, Y} f(x) \\
& \text { s.t. } \quad g(x) \leq 0 \\
& \underset{s \in S}{\vee}\left[\begin{array}{c}
Y_{i, s} \\
h_{i, s}(x) \leq 0
\end{array}\right], i=N^{\prime}+1, \ldots, N_{c} \\
& \underset{n=1, . ., N_{\max }}{\vee}\left[\begin{array}{c}
\tilde{Y}_{n} \\
\tilde{F}_{n}(x) \leq 0 \\
x_{i} \geq x_{i}^{L}, i=N^{\prime}+2, \ldots, N^{\prime}+n \\
x_{i}=0, i=N^{\prime}+n+1, \ldots, N_{c}
\end{array}\right] \\
& \Omega(Y)=\text { True } \\
& x \in\left[x^{L}, x^{U}\right] \subset \mathbb{R}^{m} \\
& Y_{i, s} \in\{\text { True, False }\}, i=N^{\prime}+1, \ldots, N_{c} ; s \in S \\
& \tilde{Y}_{n} \in\{\text { True, False }\}, n=1, \ldots, N_{\max }
\end{aligned}
$$

where the vector $h_{i, s}$ represents the constraints inside the disjunctions for the assignment of compounds and are active when compound $s$ is assigned to the $i^{t h}$ component in a mixture. $\tilde{F}_{n}$ is a vector of constraints that depend on the number of components in the mixture. Without loss of generality, the first $N^{\prime}+N_{\max }$ variables in vector $x$ are taken to be mole fractions of components in the mixture. The mole fraction, $x_{i}$, of a component $i$ is greater than a threshold value $x_{i}^{L}$ if the component is present in the mixture and is zero otherwise. The GDP formulations are converted into MINLP problems by replacing the Boolean variables with binary ones $\left(Y_{i, s}\right.$ and $\tilde{Y}_{n}$ are replaced with $y_{i, s}$ and $\tilde{y}_{n}$, respectively) and converting the logic propositions into linear inequalities. Conditional constraints inside the disjunctions are formulated using the BM and HR approaches.

Both BM and HR models have a one-to-one correspondence with the GDP formulation. The BM approach is the simplest representation of a GDP problem in a mixed-integer form. However, it introduces a large parameter $(M)$ which is often specified intuitively and can lead to poor relaxations. The HR approach, on the other hand, introduces a new set of disaggregated variables $\left(e . g ., v_{i, s}, v_{i, n}\right)$ and new constraints, increasing the size of the problem. The big-M model derived from the general GDP formulation was presented in our initial work (Jonuzaj et al., 2015), whereas the HR model is described in the following subsection.

\subsection{GDP converted into MINLP via Hull Reformulation}

In the HR formulation, the continuous variables are disaggregated into additional variables for each disjunctive term (e.g., $x_{i}$ is disaggregated into $v_{i, n}$ ). Each nonlinear disjunctive constraint, $h_{i, s}(x) \leq 0$, can be formulated using the approximation proposed by Sawaya and Grossmann (2007) in order to avoid singularities:

$$
\left((1-\varepsilon) y_{i, s}+\varepsilon\right) h_{i, s}\left(\frac{v_{i, s}}{(1-\varepsilon) y_{i, s}+\varepsilon}\right)-\varepsilon h_{i, s}(0)\left(1-y_{i, s}\right) \leq 0,
$$

where $\varepsilon$ is a small finite number (usually varying from $10^{-4}$ to $10^{-8}$ ). Hence, the resulting MINLP model derived by applying the HR approach in the mixture problem is written as follows: 


$$
\begin{aligned}
& \min _{x, y} f(x) \\
& \text { s.t. } \quad g(x) \leq 0 \\
& \left((1-\varepsilon) y_{i, s}+\varepsilon\right) h_{i, s}\left(\frac{v_{i, s}}{(1-\varepsilon) y_{i, s}+\varepsilon}\right)-\varepsilon h_{i, s}(0)\left(1-y_{i, s}\right) \leq 0, \quad i=N^{\prime}+1, \ldots, N_{c} ; s \in S \\
& x_{i}=\sum_{s=1}^{N_{s}} v_{i, s}, y_{i, s} x^{L} \leq v_{s} \leq y_{i, s} x^{U}, i=N^{\prime}+1, \ldots, N_{c} ; s \in S \\
& \left((1-\varepsilon) \tilde{y}_{n}+\varepsilon\right) \tilde{F}_{n}\left(\frac{v_{n}}{(1-\varepsilon) \tilde{y}_{n}+\varepsilon}\right)-\varepsilon \tilde{F}_{n}(0)\left(1-\tilde{y}_{n}\right) \leq 0, n=1, \ldots, N_{\max } \\
& v_{i, n} \geq v_{i, n}^{L} \tilde{y}_{n}, \quad v_{N^{\prime}+1, n} \geq v_{N^{\prime}+1, n}^{L}, \quad n=1, \ldots, N_{\text {max }} ; i=N^{\prime}+2, \ldots, N_{c} \\
& x_{i}=\sum_{n=1}^{N_{\max }} v_{i, n}, \tilde{y}_{n} x_{i}^{L} \leq v_{i, n} \leq \tilde{y}_{n} x_{i}^{U}, i=N^{\prime}+1, \ldots, N_{c} ; n=1, \ldots, N_{\max } \\
& A y \leq b \\
& x \in\left[x^{L}, x^{U}\right] \subset \mathbb{R}^{m} \\
& \tilde{y}_{n}, y_{i, s} \in\{0,1\}, \quad n=1, \ldots, N_{\max } ; i=N^{\prime}+1, \ldots, N_{c} ; s \in S
\end{aligned}
$$

where $A y \leq b$ is a set of linear inequalities resulting from the logic relations, $\Omega(Y)$. Lee and Grossmann (2003) showed that in the case of convex problems the HR bounds are at least as tight or tighter than BM.

\section{Case study: maximizing the solubility of ibuprofen}

The objective of the design problem is to identify an optimal solvent mixture in order to maximise the solubility of ibuprofen, $x_{i b u}$ (i.e., the mole fraction of ibuprofen in the mixture) at $300 \mathrm{~K}$. We have already addressed and described the problem of identifying appropriate solvents or solvent mixtures that dissolve ibuprofen (Jonuzaj et al. 2015 ) and this application is thus a suitable example to investigate the use of the proposed GDP formulations for CAM ${ }^{b} \mathrm{D}$.

\subsection{Problem description}

The problem includes phase stability and phase equilibrium relations which increase the complexity of the models. In particular, the mixture problem is highly nonlinear and nonconvex due to the solid-liquid equilibrium relations for calculating the solubility of ibuprofen, the UNIFAC model for calculating the activity coefficients and miscibility functions for every binary pair of solvent molecules, which are presented in Table 1 . In order to reduce the complexity of the model two scenarios are considered: first a simpler problem is formulated where only basic thermodynamic properties (in this case, solubility) are included in the model and then a set of highly nonlinear constraints (here, miscibility of solvent components) is added to the problem formulation. For both scenarios, the standard problem with fixed number of solvent components and the general problem, where the number of components in the mixture is bounded by an upper limit, are formulated. Hence, mixtures with one, two, three solvents $(N=1,2,3$ - restricted problem $)$ and at most three solvents $(N \leq 3$ - general problem) are designed. The solvents are selected from a list of 9 candidate compounds. The solvent molecules, the design sets and the size of the general MINLP formulations (i.e., number of equations and variables in the BM and HR models of the general problem) are also presented in Table 1 . In the restricted problem, disjunctive constraints are expressed algebraically by directly multiplying the relevant equations with the binary variables $y_{i, s}$, avoiding the use of big-M parameters (when formulating via BM) or disaggregated variables 
Table 1: Problem specifications

\begin{tabular}{|c|c|}
\hline Description & $\mathbf{N} \leq \mathbf{3}$ \\
\hline Components in mixture & ibu, $c_{1}, c_{2}, c_{3}$ \\
\hline Model Equations & $\ln \gamma_{\mathrm{ibu}}+\ln \gamma_{\mathrm{ibu}}=\frac{\Delta H_{f u s}}{R}\left[\frac{1}{T_{m}}-\frac{1}{T}\right], \frac{\partial \ln \gamma_{i j}}{\partial x_{i}^{i, j}}+\frac{1}{x_{i}^{i, j}} \geq 0$, UNIFAC Model \\
\hline Candidate solvents & $\begin{array}{c}\text { Acetone, Chloroform (chlorof), Ethanol, Ethyl-acetate, } \\
\text { Methanol (meth), MIBK, 2-Propanol, Toluene, Water }\end{array}$ \\
\hline $\begin{array}{c}\text { Size of general MINLP } \\
\text { (all properties included) }\end{array}$ & $\begin{array}{c}\text { BM: 594 equations, 72 discrete variables, 361 continuous variables } \\
\text { HR: 703 equations, 72 discrete variables, 545 continuous variables }\end{array}$ \\
\hline $\begin{array}{c}\text { Size of general MINLP } \\
\text { (miscibility excluded) }\end{array}$ & $\begin{array}{c}\text { BM: 241 equations, 72 discrete variables, 60 continuous variables } \\
\text { HR: 272 equations, 72 discrete variables, 87 continuous variables }\end{array}$ \\
\hline
\end{tabular}

(when formulating via HR) in the MINLP model. Therefore, both BM and HR models result in the same MINLP formulations for the restricted model.

\subsection{Results and discussion}

All models were implemented and solved in GAMS version 24.2.3 and were run on a single core of a dual 6 core Intel Xeon X5675 machine at $3.07 \mathrm{GHz}$ with 48GB of memory. Several global solvers were tested but did not reach convergence, and DICOPT, a local MINLP solver, was used to solve the problems. Due to the highly nonlinear nature of the equations and the size of the models (see Table 11, multiple initial guesses were used and several local solutions were identified. The best solutions obtained from all the runs with different starting points, in the first (i.e., without the miscibility constraints) and in the second scenario (i.e., with the miscibility constraints) are summarised in Tables 2 and 3 respectively. The highest solubility is achieved in a mixture of two solvents. A mixture of chloroform and water is identified as the optimal solvent mixture when the miscibility functions are not included in the formulations and a binary mixture of chloroform and methanol is identified as optimal when the miscibility constraints are added.

In both scenarios, the CPU time increases rapidly with the number of components, due to the increased size and complexity of the problems. HR formulations of the general problem require less CPU time than BM formulations. In the second scenario, HR appears to be more effective than solving the highly nonlinear problem with a fixed number of mixture components several times $(N=1,2,3)$, from the perspective of computational cost.

Table 2: Results of BM (Jonuzaj et al., 2015) and HR formulations without miscibility constraints.

\begin{tabular}{c|cccccccc}
\hline \hline Case & $x_{\text {ibu }}$ & $c_{1}$ & $x_{c_{1}}$ & $c_{2}$ & $x_{c_{2}}$ & $c_{3}$ & $x_{c_{3}}$ & CPU(s) \\
\hline $\mathrm{N}=1$ & 0.31833 & chlorof & 0.68167 & & & & & 0.35 \\
$\mathrm{~N}=2$ & $\mathbf{0 . 3 4 9 2 8}$ & chlorof & 0.49706 & water & 0.15366 & & & 5.02 \\
$\mathrm{~N}=3$ & 0.34915 & chlorof & 0.49691 & water & 0.15294 & meth & 0.00100 & 114.49 \\
\hline $\mathrm{N} \leq 3(\mathrm{BM})$ & $\mathbf{0 . 3 4 9 2 8}$ & chlorof & 0.49706 & water & 0.15366 & & & 316.92 \\
$\mathrm{~N} \leq 3(\mathrm{HR})$ & $\mathbf{0 . 3 4 9 2 8}$ & chlorof & 0.49706 & water & 0.15366 & & & 223.89 \\
\hline
\end{tabular}

\section{Conclusions}

A general methodology for designing optimal mixtures using GDP within the $\mathrm{CAM}^{\mathrm{b}} \mathrm{D}$ framework has been presented. Employing GDP techniques to formulate the discrete choices inherent in the mixture design problem can facilitate the problem formulation, making it possible to optimise simultaneously the number, identities and compositions of components in the mixture. Both 
Table 3: Results of BM (Jonuzaj et al. 2015) and HR formulations with miscibility constraints.

\begin{tabular}{c|cccccccc}
\hline \hline Case & $x_{\text {ibu }}$ & $c_{1}$ & $x_{c_{1}}$ & $c_{2}$ & $x_{c_{2}}$ & $c_{3}$ & $x_{c_{3}}$ & CPU(s) \\
\hline $\mathrm{N}=2$ & $\mathbf{0 . 3 3 3 8 3}$ & chlorof & 0.52292 & meth & 0.14325 & & & 4.87 \\
$\mathrm{~N}=3$ & 0.33375 & chlorof & 0.52263 & meth & 0.14262 & ethanol & 0.00100 & 107.90 \\
\hline $\mathrm{N} \leq 3(\mathrm{BM})$ & $\mathbf{0 . 3 3 3 8 3}$ & chlorof & 0.52292 & meth & 0.14325 & & & 369.06 \\
$\mathrm{~N} \leq 3(\mathrm{HR})$ & $\mathbf{0 . 3 3 3 8 3}$ & chlorof & 0.52292 & meth & 0.14325 & & & 93.59 \\
\hline
\end{tabular}

BM and HR approaches can be applied successfully to convert the GDP mixture formulations into MINLP problems. In the example studied, the HR technique results in more computationally efficient solutions than the BM approach. Furthermore mixtures outperform pure solvents, showing the benefit of using mixtures instead of pure compounds to attain enhanced behaviour. This promising framework is currently being applied to additional examples, so that the performance of the proposed methodology can be further investigated.

Acknowledgments: The authors gratefully acknowledge financial support from the Engineering and Physical Sciences Research Council (EPSRC) of the UK (EP/J003840/1).

Data statement: Data underlying this article can be accessed on Zenodo at http://dx.doi. org/10.5281/zenodo.45297, and used under the Creative Commons Attribution license.

\section{References}

L. E. K. Achenie, R. Gani, V. Venkatasubramanian, 2003. Computer aided molecular design: theory and practice. Elsevier, Amsterdam, The Netherlands.

A. Buxton, A. G. Livingston, E. N. Pistikopoulos, 1999. Optimal design of solvent blends for environmental impact minimization. AIChE Journal 45, 817-843.

A. Duvedi, L. E. K. Achenie, 1997. On the design of environmentally benign refrigerant mixtures: a mathematical programming approach. Computers \& Chemical Engineering 21, 915-923.

R. Gani, 2004. Chemical product design: challenges and opportunities. Computers \& Chemical Engineering 28, 24412457.

I. E. Grossmann, F. Trespalacios, 2013. Systematic modeling of discrete-continuous optimization models through generalized disjunctive programming. AIChE Journal 59, 3276-3295.

S. Jonuzaj, P. T. Akula, P. M. Kleniati, C. S. Adjiman, 2015. The formulation of optimal mixtures with Generalized Disjunctive Programming: A solvent design case study. AIChE Journal. DOI: 10.1002/aic.15122.

A. T. Karunanithi, L. E. K. Achenie, R. Gani, 2005. A new decomposition-based computer-aided molecular/mixture design methodology for the design of optimal solvents and solvent mixtures. Industrial \& Engineering Chemistry Research 44, 4785-4797.

J. A. Klein, D. T. Wu, R. Gani, 1992. Computer aided mixture design with specified property constraints. Computers \& Chemical Engineering 16, S229-S236.

S. Lee, I. E. Grossmann, 2003. Generalized convex disjunctive programming: Nonlinear convex hull relaxation. Computational Optimization and Applications 26, 83-100.

A. I. Papadopoulos, M. Stijepovic, P. Linke, P. Seferlis, S. Voutetakis, 2013. Toward optimum working fluid mixtures for organic Rankine cycles using molecular design and sensitivity analysis. Industrial \& Engineering Chemistry Research 52, 12116-12133.

R. Raman, I. E. Grossmann, 1994. Modelling and computational techniques for logic based integer programming. Computers \& Chemical Engineering 18, 563-578.

N. W. Sawaya, I. E. Grossmann, 2007. Computational implementation of non-linear convex hull reformulation. Computers \& Chemical Engineering 31, 856-866.

E. Siougkrou, A. Galindo, C. S. Adjiman, 2014. On the optimal design of gas-expanded liquids based on process performance. Chemical Engineering Science 115, 19-30.

C. C. Solvason, N. G. Chemmangattuvalappil, F. T. Eljack, M. R. Eden, 2009. Efficient visual mixture design of experiments using property clustering techniques. Industrial \& Engineering Chemistry Research 48, 2245-2256.

R. Vaidyanathan, M. El-Halwagi, 1996. Computer-aided synthesis of polymers and blends with target properties. Industrial \& Engineering Chemistry Research 35, 627-634.

N. A. Yunus, K. V. Gernaey, J. M. Woodley, R. Gani, 2014. A systematic methodology for design of tailor-made blended products. Computers \& Chemical Engineering 66, 201-213. 